Relations industrielles

Industrial Relations

\title{
La légalité des clauses de sécurité syndicale
}

\section{Louis-Philippe Pigeon}

Volume 4, numéro 10, juin 1949

URI : https://id.erudit.org/iderudit/1023544ar

DOI : https://doi.org/10.7202/1023544ar

Aller au sommaire du numéro

Éditeur(s)

Département des relations industrielles de l'Université Laval

ISSN

0034-379X (imprimé)

1703-8138 (numérique)

Découvrir la revue

Citer cet article

Pigeon, L.-P. (1949). La légalité des clauses de sécurité syndicale. Relations industrielles / Industrial Relations, 4(10), 91-94.

https://doi.org/10.7202/1023544ar

Tous droits réservés (C Département des relations industrielles de l’Université Laval, 1949
Ce document est protégé par la loi sur le droit d'auteur. L'utilisation des services d'Érudit (y compris la reproduction) est assujettie à sa politique d'utilisation que vous pouvez consulter en ligne.

https://apropos.erudit.org/fr/usagers/politique-dutilisation/ 


\section{Bulletin des relations industrielles}

Volume 4, numéro 10

QUÉBEC

Juin 1949

Publié par le

Département des relations industrielles, Faculté des sciences sociales, Université Laval.

Georges-Henti Lévesque, o.p., doyen GÉRARD TREMBLAY, directeur GÉRARD Dion, sous-directeur JEAN GaGne, secrétaire

CharLes BÉlanger, administrateur

Le Bulletin paraît mensuellement de septembre à juin (dix numéros par année). Abonnement annuel: Canada: \$2.00; étranger: $\$ 2.50$. Vingt-cinq cents le numéro. Publication répertoríé dans le "Canadian Index".

Adressez toute correspondance au secrétaire de rédaction

Géraro Dion

2, rue de l'Université, Québec.

Bulletin des relations industrielles

Volume 4, numéro $10 \quad$ Juin 1949

\section{Sommaire}

La légalité des clauses de sécurité sundicale,

Louis-Philippe Pigeon

Les métiers qui meurent dans

l'industrie du bâtiment,

Marcel Clément

L'économie doit être placée au service de l'homme,

Paul-H. Plamondon.

Visée d'avenir sur la convention collective,

Jacques Archambault

Index pour 1948-1949

\section{Collaborateurs}

Archambault, Jacques, B.A., M.Sc.S., secrétaire du Conseil central des syndicats nationaux catholiques de Joliette.

Clément, Marcel, professeur, Faculté des sciences sociales de Laval.

Pigeon, Louis-Philippe, c.r., avocat au Barreau de Québec et professeur aux $\mathrm{Fa}$ cultés de droit et des sciences sociales de Laval.

Plamondon, Paul-H., président de la Chambre de Commerce de Québec, propriétaire de H. Thivierge le Costumier Enr., Québec.

Autorisé comme envoi postal de deuxième classe, ministère des postes, Ottawa, Canada.

\section{LA LÉGALITÉ DES CLAUSES DE SÉCURITÉ, SYNDICALE}

\author{
Ls-Philippe Pigeon
}

\section{Ce qui rend difficile le problème}

La difficulté de la question provient de ce qu'elle est au fond un des aspects du problème de la liberté juridique.

Le premier principe du droit peut s'énoncer comme suit: «'out ce qui n'est pas défendu est permis. » Tout en étant simple, ce principe est très difficile d'application. La liberté en effet, ne se conçoit pas sans des restrictions parce qu'il faut toujours empêcher que la liberté des uns ne détruise la liberté des autres.

Les solutions de liberté sont donc difficiles, compliquées. Dans les régimes d'autorité, le problème de la liberté ne se pose pas: il faut obéir aux «ordres» donnés. Rien ne limite les «ordres». Quand on croit à la liberté, on ne peut se contenter d'y croire, il faut entreprendre de la définir. Donc prohiber certains actes notamment ceux qui portent préjudice à autrui. Qu'est-ce qui porte préjudice à autrui, ce n'est pas simple du tout. Il y a très peu d'activités qui ne causent pas un certain préjudice, alors on est obligé de définir ce qu'on entend par préjudice. Ainsi dans le domaine des rapports entre propriétaires d'immeubles voisins on dira que l'on doit subir les inconvénients ordinaires comme les cheminées usuelles.

La liberté n'étant pas absolue, par conséquent la liberté d'association ne peut pas signifier que les associations ouvrières peuvent réclamer n'importe quelle clause de sécurité syndicale. Il doit y avoir une limitation quelconque. La loi Taft-Hartley est apparue comme une réaction justifiable à l'encontre de ce que le Wagner Act avait de faux, fondé comme il l'était sur une liberté illimitée pour les syndicats ne définissant que des droits pour les unions et que des devoirs pour les patrons. Il ne faut pas oublier qu'aux EtatsUnis nombre d'unions ouvrières prétendent, dans leurs constitutions, se placer au-dessus de la loi, s'ériger en seuls juges de ce qui doit leur être permis, de là leur prétention à se soustraire à l'obéissance aux injonctions des tribunaux.

Dans le domaine de la sécurité syndicale, ce qui fait le problème, c'est que d'une part on revendique des droits ab-

$\bar{N}$. de la $R$.-Nous publions ici le résumé d'un cours donné à la Faculté des sciences sociales. 
solus pour les unions, et d'autre part certains employeurs se refusent à toute forme de sécurité syndicale. La justice est cependant dans le juste milieu.

Il faut que l'ouvrier ne soit pas asservi au syndicat. D'un autre côté, il est raisonnable que l'on réclame un certain degré de coercition ayant pour objet d'empêcher que l'association ouvrière soit toujours obligée de combattre pour maintenir son existence.

\section{Législation provinciale}

Les apinions sont très partagées sur ce que notre législation permet. La question n'a pas été tranchée par les tribunaux de droit commun, elle n'a été étudiée que dans des sentences arbitrales ou des articles de revue.

A mon avis, la loi provinciale actuelle n'interdit aucune forme de sécurité syndicale. Il y a des hommes de loi qui n'acceptent pas cela, mais après avoir étudié leurs arguments, je ne crois pas que leurs objections soient fondées sauf quand il s'agit d'ouvriers régis par une ordonnance de salaire minimum. Ces ordonnances ont pour résultat d'interdire toute déduction qui aurait pour effet d'abaisser le salaire en bas du minimum fixé par l'ordonnance, par conséquent une clause de sécurité syndicale qui comporterait une déduction de ce genre, serait invalide. Ce cas ne peut se présenter que très exceptionnellement, car les conventions collectives prévoient ordinairement un salaire plus élevé que le minimum.

D'après notre loi provinciale, il n'y a pas d'autres restrictions. La clause contre laquelle on peut formuler les plus forts arguments juridiques est l'atelier syndical, laquelle oblige un nouvel embauché à entrer dans le syndicat sous peine de perdre son emploi. Raison: La Loi des relations ouvrières défend à quelqu'un d'user de menaces pour amener un ouvrier à devenir membre d'un syndicat. Toutefois, cette même loi maintient par ailleurs le droit de congédiement de l'employeur.

1. Atelier fermé.-Aucun argument fondé sur un texte de loi ne peut être invoqué contre une telle formule. L'employeur est libre d'embaucher qui lui plaît. L'ouvrier qui n'est pas encore à l'emploi de l'employeur n'a pas un droit légal à être embauché. Alors l'employeur a sûrement le droit de ne pas embaucher un travailleur qui n'est pas membre du syndicat.

Les textes qui concernent l'embauchage ne parlent que du refus d'emploi à raison de ce que l'on est membre ou officier d'une association. La clause d'atelier fermé est donc juridiquement licite. Aucun doute sur sa légalité. Le texte qui touche les distinctions dans l'embauchage ne dit pas «paree qu'il est membre ou parce qu'il n'est pas membre». Ce texte qui existait auparavant a été abrogé. Cela montre bien que l'intention du législateur n'était pas de prohiber une telle clause. Cela étant, comment pourrait-on rendre illégale l'autre clause qui est beaucoup moins stricte; l'atelier syndical ? Par ailleurs au point de vue économique la clause d'atelier fermé peut présenter de graves dangers pour l'employeur. Sauf dans certaines industries v.g. construction, où l'on est en présence d'unions qui ont un grand nombre de membres, un réservoir de main-d'oeuvre, le droit de gestion peut se trouver restreint considérablement par l'application d'une telle clause.

2. Atelier syndical.-Nous venons de voir que d'après la Loi des relations ouvrières, l'atelier fermé est indiscutablement permis. Cela pourrait à la rigueur, suffire à disposer pratiquement de tout argument contre la licéité juridique de l'atelier syndical et spécialement de l'atelier syndical imparfait. En effet, si l'on peut légalement réclamer de l'employeur l'atelier fermé, ce dernier n'a pas d'intérêt à contester la légalité d'une clause beaucoup moins rigoureuse.

Il y a toutefois une objection juridique à considérer surtout pour l'atelier syndical parfait à cause de son application aux employés déjà au service de l'entreprise. Cette objection vient du texte de l'article 22 de la Loi des relations ouvrières défendant d'user «d'intimidation ou de menaces pour amener quiconque à devenir membre d'une association. » L'employeur n'use-t-il pas de menaces envers ses employés en souscrivant cette clause ? Je ne le pense pas. Au point de vue juridique, l'ouvrier n'a pas droit à son emplei au-delà de la période qui le lie à son employeur. Dans l'industrie, les emplois sont ordinairement à lheure. Alors, le contrat est résiliable à chaque heure. L'employeur en effectuant un congédiement, use d'un droit certain et n'a pas à rendre compte de ses motifs sauf toujours le cas de congédiement pour activité syndicale. Ici, il ne s'agit pas d'activité, mais d'inactivité. La clause n'oblige l'employeur à aucune menace. Il peut s'y conformer en n'en faisant absolument aucune. S'il y a lieu, il fera le congédiement, rien d'autre ne lui est demandé.

L'employé peut incontestablement quitter son emploi sans donner aucune raison. L'employeur peut de même résilier le contrat de travail. Il est un principe bien reconnu en droit civil que l'exercice d'un droit n'est pas une me- 
nace. Ainsi quoique l'on ait le droit de faire résilier un contrat consenti sous une menace, un débiteur ne peut se plaindre des garanties onéreuses qu'il a concédées sous la pression de poursuites judiciaires. De même un patron ne peut attaquer un contrat collecifif obtenu par ses ouvriers usant du diroit de grève.

Conclusion: même la clause d'atelier syndical parfait est valide. Et cela implique la validité de toutes les autres clauses de sécurité syndicale. Cependant, la formule Rand ou retenue syndicale obligatoire exige une considération particulière quoique l'atelier syndical parfait ou l'atelier fermé impliquent davantage.

3. Formule Rand ou retenue syndicale obligatoire.-Ici, il faut tenir compte d'une autre objection, savoir si une telle clause est une « condition de travail». A mon avis cette expression comprend tout ce qui concerne les relations entre em ployeur et salarié. On invoque la décision dans la cause Lazarovitz vs Cour des Sessions (69 B.R. 214) qui avait trait à des prix à charger au public. Le principe appliqué en cette cause est le suivant: «Si généreux que soient les termes dont on s'est servi, ils se restreignent à ce sur quoi on a voulu statuer.» Dans la Loi de la convention collective, l'expression «toutes autres dispositions» interprétée littéralement aurait voulu dire n'importe quoi. Alors, la Cour d'Appel a dit: « La loi s'est préoccupée d'une seule chose: rapports entre employeurs et salariés. Conséquemment, le champ des décrets en exécution de cette loi doit être restreint aux clauses «ejusdem generis» avec l'énumération, soit des clauses qui concernent les rapports entre employeurs et salariés. » C'est ce même principe qu'il faut appliquer à la Loi des relations ouvrières, et il conduit à dire que tout ce qui concerne des relations entre employeurs et salariés peut faire l'objet de la convention collective.

En France, la même expression «conditions de travail $\gg$ selon l'opinion unanime comprend toutes les relations entre employeurs et salariés y compris les déductions sur le salaire.

Rien ne s'oppose donc à la validité des clauses de retenue de cotisations syndicales. S'il s'agit de déductions volontaires on aura de véritables transports de créance qui ne présentent vraiment aucune difficulté. On peut se demander si, même en l'absence de stipulation dans la convention l'employeur pourrait les refuser; il ne pourrait le faire qu'en congédiant les signataires; ne seraitce pas un renvoi pour activité syndicale?

Quand il s'agit d'une retenue obligatoire on allègue souvent que l'employeur n'a pas le droit de «taxer» ses ouvriers. Notons d'abord que personne ne formule cette objection quand il s'agit par exemple de contributions obligatoires à des pensions de retraite. En droit on n'oblige pas les travailleurs à accepter la déduction, mais s'ils ne le font pas, ils n'ont qu'à s'en aller. La convention collective ne supprime pas les conventions individuelles. Celles-ci demeurent et gardent leurs termes propres et ce sont elles qui fixent la durée de l'emploi. L'usage universel est que ces contrats sont à court terme. Le contrat est résiliable à la volonté des deux parties. Ce droit de résiliation permet à l'employeur d'établir un règlement qui stipule tout ce qui n'est pas immoral. L'ouvrier est libre de refuser en quittant son emploi, autrement, il accepte les conditions fixées. C'est là d'ailleurs ce qui rend nécessaire l'organisation syndicale.

Comme je l'ai déjà signalé, une ordonnance de salaire minimum peut rendre illégale la retenue syndicale dans des cas exceptionnels. Un décret pourrait aussi avoir le même effet, par exemple un décret qui stipule que les salaires supérieurs doivent être maintenus.

On peut tout résumer la situation juridique en disant que la Loi des relations ouvrières n'a pas défini la liberté d'association autrement que le droit de faire partie d'une association et n'a pas parlé d'un droit de n'en pas faire partie.

\section{Législation fédérale}

Les dispositions relatives à la sécurité syndicale se retrouvent dans la Loi sur les relations industrielles et sur les enquêtes visant les différends du travail (Bill 195).

Cette loi comporte deux dispositions qui se rapportent à ce sujet.

Tout d'abord elle déclare expressément que rien n'empêche d'insérer dans une convention collective une disposition exigeant comme condition d'emploi, que l'on soit membre d'une union ouvrière déterminée ou accordant préférence dans l'embauchage aux membres d'une union déterminée. Donc toute clause de sécurité syndicale est licite.

En second lieu, il y a une restriction: aucune condition ne sera permise dans une convention collective requérant un employeur de renvoyer un salarié parce qu'il est membre d'une union autre que d'une union déterminée. Je pense que ceci a pour résultat d'empêcher l'application de la clause d'atelier fermé, quand un travailleur est expulsé d'un syndicat parce qu'il a adhéré à une union rivale. Conséquence, si l'employé n'est pas 
encore embauché, on ne fait que l'empêcher d'obtenir un emploi et rien n'empêche la clause d'avoir son effet, mais s'il est déjà au service de l'entreprise, l'union ne peut alors exiger le renvoi.

La loi fédérale a donc voulu permettre ex- pressément les clauses de sécurité syndicale tout en sauvegardant le droit des salariés de quitter une union jouissant de l'atelier fermé, pour adhérer à une autre association destinée à la supplanter comme agence de négociation collective.

\section{LES MÉTIERS QUI MEURENT DANS L'INDUSTRIE DU BÂTIMENT}

\section{MARCEL CléMENT}

Au cours de l'enquête que nous avons poursuivie sur la situation de la main-d'oeuvre dans l'industrie du bâtiment de la province de Québec, nous avons été amenés à enregistrer deux phénomènes particulièrement nets, et dont les conséquences, à longue échéance, risquent, si l'on n'y porte un prompt remède, d'être fort onéreuses. D'une part, il est apparu que certaines catégories de métiers jouissaient parmi les jeunes, d'un succès croissant et incontrôlé, et d'autre part, certaines autres catégories sont désertées à un rythme inquiétant.

Le tableau I ci-dessous indique l'effectif, par groupe d'âge, des apprenitis et des salariés qualifiés des huit principaux métiers de l'Industrie du bâtiment, tel qu'il a été révélé par un questionnaire rempli à la date du 31 juillet 1948. On tiendra compte de ce que ces chiffres:

a) ne recouvrent ni les contracteurs, ni les artisans installés à leur compte, ni les journaliers, mais exclusivement les salariés qualifiés des métiers intéressés;

b) ne concernent que les régions contrôlées par un comité paritaire.

TABLEAU 1: EFFECTIF ETABLI PAR GROUPE D'AGE, DES APPRENTIS ET DES SALARIES QUALIFIES DES HUIT PRINCIPAUX METIERS DE L'INDUSTRIE DU BATIMENT DANS LA PROVINCE DE QUEBEC, AU 31 JUILLET 1949

\begin{tabular}{|c|c|c|c|c|c|c|c|}
\hline $\begin{array}{c}\text { Nomenclature } \\
\text { des } \\
\text { métiers }\end{array}$ & $\begin{array}{l}\text { Effectif } \\
\text { des } \\
\text { apprentis }\end{array}$ & $\begin{array}{l}\text { Salariés } \\
\text { ayant } \\
\text { moins de } \\
25 \text { ans }\end{array}$ & $\begin{array}{c}\text { Salariés } \\
\text { ayant } \\
\text { de } 25 \text { à } \\
35 \text { ans }\end{array}$ & $\begin{array}{c}\text { Salariés } \\
\text { ayant } \\
\text { de } 35 \text { à } \\
45 \text { ans }\end{array}$ & $\begin{array}{l}\text { Salariés } \\
\text { ayant } \\
\text { de } 45 \text { à } \\
55 \text { ans }\end{array}$ & $\begin{array}{c}\text { Salariés } \\
\text { ayant } \\
\text { plus de } \\
55 \text { ans }\end{array}$ & $\begin{array}{c}\text { Total des } \\
\text { salariés } \\
\text { employés } \\
\text { dans chaque } \\
\text { métier } \\
\end{array}$ \\
\hline Charpentiers-menuisiers & 1,529 & 795 & 2,561 & 2,711 & 1,919 & 1,231 & 10,746 \\
\hline Briqueteurs & 367 & 199 & 477 & 452 & 517 & 242 & 2,254 \\
\hline Plâtriers-maçons & 308 & 74 & 201 & 381 & 320 & 158 & 1,442 \\
\hline Peintres & 706 & 432 & 1,156 & 1,522 & 818 & 336 & 4,970 \\
\hline Plombiers & 2,321 & 487 & 780 & 727 & 326 & 187 & 4,828 \\
\hline $\begin{array}{l}\text { Poseurs d'appareils } \\
\text { de chauffage }\end{array}$ & 378 & 82 & 134 & 95 & 54 & 20 & 763 \\
\hline Ferblantiers & 271 & 59 & 227 & 193 & 132 & 65 & 947 \\
\hline Electriciens & 1,557 & 614 & 795 & 472 & 242 & 35 & 3,715 \\
\hline $\begin{array}{l}\text { Total des salariés établi } \\
\text { par groupe d'âge }\end{array}$ & 7,437 & 2,742 & 6,331 & 6,553 & 4,328 & 2,274 & 29,665 \\
\hline
\end{tabular}

